American Journal of Biochemistry and Biotechnology 7 (2): 84-89, 2011

ISSN 1553-3468

(C) 2011 N.E. Ibrahim et al., This open access article is distributed under a Creative Commons Attribution

(CC-BY) 3.0 license

\title{
Biochemical Studies on Recombinant Human Isobutyryl-CoA Dehydrogenase
}

\author{
${ }^{1}$ Nasser E. Ibrahim and ${ }^{2} \mathrm{Al}-$ Walid A. Mohsen \\ ${ }^{1}$ Department of the Bioinformatics Genetic Engineering and Biotechnology, \\ Research Institute, Minufiya University, Sadat Branch, Sadat City, Egypt \\ ${ }^{2}$ University of the Pittsburgh, Children's Hospital of Pittsburgh of UPMC, PN, USA
}

\begin{abstract}
Problem statement: Human Isobutyryl-CoA Dehydrogenase (IBD) is member of the Acyl-CoA Dehydrogenases family. It is involved in Val metabolism. In this study we modified the purification method of the IBD and further spectroscopic characterization was conducted. Approach: Using DTT in the buffers during the purification, IBD purified to homogeneity by different chromatographic steps. Reactivity of Cys residues were measured using thiol modifying agent DNTB. Results: IBD found to be homotetramer with $42.7 \mathrm{KDa}$ for each subunit and the value of its pI was 6.2. Conclusion: Thiol modifying reagent showed crucial role (s) of some Cys residues for IBD structure, FAD binding and/or activity.
\end{abstract}

Key words: Acyl-CoA dehydrogenase, isobutyryl-CoA dehydrogenase, valine degradation, Sudden Infant Death Syndrome (SIDS), cys residues, recombinant human, ferricenium assay, jamaican vomiting, thiol modifying

\section{INTRODUCTION}

Acyl-CoA Dehydrogenases (ACADs) form a family of ten members that catalyze the a, $\cap$ desaturation of acyl-CoA thioesters to corresponding trans-2-enoyl-CoA. They are assumed to share the same chemical mechanism, however, they differ in their specificity for different types of fatty acids linked to CoA (Ye et al., 2004). This family can be differentiated into two subclasses. First subclass comprises five members Very long-chain acyl-CoA dehydrogenase1 and 2 (VLCAD1, VLCAD2), Longchain acyl-CoA dehydrogenase (LCAD), Mediumchain acyl-CoA dehydrogenase (MCAD) and Shortchain acyl-CoA dehydrogenase (SCAD) that utilizing "straight chain", substrates, that in turn, enable the sequential degradation of the substrates in $\beta$-oxidation cycle (Ye et al., 2004). The second subclass encompasses the enzymes involved in degradation of "branched chain" acyl-CoA thioesters arising from amino acid catabolism. These include Isovaleryl-CoA dehydrogenase (IVD), Short-Branched Chain-CoA Dehydrogenase (SBCAD), Glutaryl-CoA Dehydrogenase (GCAD) and a newer member Isobutyryl-CoA dehydrogenase or IBD. With the exception of VLCAD1 (VLCAD2 not characterized yet) that exists as dimer, the ACADs are found in homotetrameric form with one molecule of Flavin
Adenine Dinucleotide (FAD) non-covalently bound to each monomer (Izai et al., 1992). The tenth members ACAD10 is not characterized yet (Ye et al., 2004) (Ye et al., 2004).

Metabolic disorders due to inherited genetic mutations in ACAD genes have two pathological consequences. One is the result of energy deficiency and shortage of substrates for gluconeogensis (generation of accessible glucose supply) due to reduction of the flux through the fatty acid oxidation pathway. The second is the toxic effect of intermediate metabolites that accumulate due to enzyme deficiency. Effects of the latter are the most serious and can cause serious neurological damage. The net result is crisis situations in metabolic decompensation and clinical disease, which may be fatal especially in infants. That in some cases leads to the Sudden Infant Death Syndrome (SIDS) (Eminoglu et al., 2011).

cDNA exhibiting sequence homology with ACAD family was initially isolated from human adult brain and skin fibroblasts as a precursor and was designated as ACAD8 (Telford et al., 1999).

Preliminary activity profiles obtained with heterologously expressed enzyme suggested that the protein was involved in the catabolism of branched chain amino acids which was with the occurrence of specific genetic defects and their physiological consequences (Roe et al., 1998; Sass et al., 2004). It

Corresponding Author: Nasser E. Ibrahim, Department of the Bioinformatics Genetic Engineering and Biotechnology, Research Institute, Minufiya University, Sadat Branch, Sadat City, Egypt 
was thus deduced that the enzyme was involved in Val catabolism and specifically in the dehydrogenation of isobutyryl-CoA. Accordingly the enzyme was named isobutyryl-CoA dehydrogenase (IBD) (Sass et al., 2004). Recently, different inherited mutations of ACAD8 have been detected and follow-up algorithm diagnosis of IBD deficiency has been reported (Oglesbee et al., 2007). Earlier studies had assigned isobutyryl-CoA degradation activity to SBCAD, as in case of rat SBCAD which can utilize both 2-methylbutyryl-CoA and isobutyryl-CoA that emerged from Ile and Val degradation, respectively (Willard et al., 1996; Battaile et al., 2004).

IBD crystallographic structure data indicated that Glu-376 is the catalytic base and that the substratebinding cavity is shorter and wider relative to SCAD, which allows the optimal binding of the isobutyryl-CoA substrate. Another difference between IBD structure and other ACAD is that the conserved Tyr or Phe that defined side of the binding cavity is replaced by Leu residue (Leu-375). In comparison with IVD, the lateral expansion of the binding cavity is not observed in IBD (Battaile et al., 2004). Here, we describe modified purification method for recombinant human IBD and studies on its biochemical characters.

\section{MATERIALS AND METHODS}

Q-Sepharose FF and Superdex 200 prep grade HiLoad 26/60 (Amersham Biosciences, GE). Hydroxyapatite CHT10-I Ceramic (Bio-Rad). Hydroxyapatite (Fluka). Ferricenium hexafluorophosphate (Aldrich). Cleland's reagent (DTT), Ellman's reagent and all other chemicals are from Sigma.

Expression and purification of recombinant human IBD: Recombinant IBD was expressed and purified as previously mentioned (Willard et al., 1996) with some modifications. The modification includes using of 5 ( M DTT in all buffers, using ammonium sulfate precipitations and two hydroxyapatite columns following the Q-Sepharose one.

Molecular weight determination: IBD molecular weight was determined by MALDI-TOF (Bruker Biflex time-of- flight mass spectrometer, Bruker Daltonik, Bremen, Germany) equipped with a 26-sample SCOUT source, a nitrogen UV laser $\left(L_{\max }=337 \mathrm{~nm}\right)$ and a delayed extraction system. A concentrated sample of IBD, $1 \mu \mathrm{l}\left(\approx 7 \mathrm{mg} \mathrm{mL}^{-1}\right)$, was mixed with $1 \mu \mathrm{L}$ matrix solution (super saturated a-cyano-4-hydroxycinnamic acid in acetonitrile: $0.1 \%$ trifluoroacetic acid in water, 2: 1) on the MALDI-TOF target and allowed to dry at room temperature (Mann and Talbo, 1996). IBD native molecular weight was estimated by gel filtration (Superdex 200, 25 mM KPi, pH 7.8, 150 mM KCl, 5\% Glycerol, 5 (M DTT).

Isoelectric focusing: ProteoGel IPG strips $(7 \mathrm{~cm}$, Sigma) with $\mathrm{pH}$ range 5-8 and IPGphor IEF system (Amersham Biosciences, GE) were used. A lyophilized sample, from $150-250 \mu \mathrm{L}$ of $4 \mu \mathrm{M}$ IBD was redissolved in rehydration buffer (2D-Manual, Amersham Biosciences, GE) and used for rehydration of the strip for $15 \mathrm{~h}$. After IEF, the strip was equilibrated in the equilibration buffer containing DTT for $1 \mathrm{~h}$. and then applied to an SDS-PAGE gel. IBD was detected by chemiluminescence using antibody specific for Histagged IBD kindly provided by Dr. P. Bross (Aarhus, Denmark).

Spectroscopic methods: Visible and ultraviolet spectra were recorded with a Kontron Uvikon 930 spectrophotometer. Anaerobic reactions were followed in Thunberg type cuvettes equipped with two side arms. Oxygen depletion was achieved by at least four flushing and evacuation cycles using $\mathrm{O}_{2}$-free argon in the presence of an oxygen scavenging system $(0.1 \mu \mathrm{M}$ glucose oxidase, $0.1 \mathrm{M}$ glucose and $0.1 \mu \mathrm{M}$ catalase enzyme) in a sidearm of the cuvette. Rapid reaction studies were conducted with a stopped-flow spectrophotometer with $1.0 \mathrm{~cm}$ path length cell and a diode array detector ( $\mathrm{J}$ and $\mathrm{M}$ Aalen), using BioKine. The fastest acquisition time of 1 spectrum $/ 0.7 \mathrm{~ms}$ in the range of $300-650 \mathrm{~nm}$. Unless otherwise stated all spectrophotometer measurements were carried out at $25^{\circ} \mathrm{C}$ in $40 \mathrm{mM}$ potassium phosphate buffer, $\mathrm{pH} 7.8$, containing 5\% glycerol. Acyl-CoA solutions were standardized by spectrophotometer in $10 \mathrm{mM} \mathrm{KPi}$, pH 6.0 using $\sum_{260}=15.4 \mathrm{mM}^{-1} \mathrm{~cm}^{-1}$. Absorption coefficients for IBD were determined using the SDS method and $\sum_{450}=11.3 \mathrm{mM}^{-1} \cdot \mathrm{cm}^{-1}$ for free FAD (Mayhew and Massey, 1969; Whitby, 1953).

Enzyme assays and pH-dependence of activities: Activities were measured using the ferricenium assay (Lehman et al., 1990). Measurements of $\mathrm{pH}$ dependences were carried out in $40 \mathrm{mM}$ buffer containing $5 \%$ glycerol and $150 \mathrm{mM} \mathrm{KCl}$ at the indicated $\mathrm{pH}$ values and at $25^{\circ} \mathrm{C}$. IBD concentration was $\approx 20 \mathrm{nM}$ and the buffers used were: $\mathrm{pH} 5.0$ : acetate/KOH; pH 5.5: MES/KOH; pH 6.0-6.5: HEPES/KOH; pH 7.0-7.5: MOPS/KOH; pH 8.0: Tris/HCL; pH 8.5-9.0: Tricine/KOH; pH 9.5-10.0: Glycine/KOH. For anaerobic measurements, oxygen was removed from enzyme samples in Thunberg type cuvetts by 5-6 cycles of flushing with oxygen-free argon/vacuum. 
Reactivity of cys thiol groups: IBD, 1 / $\mathrm{M}$ in $40 \mathrm{mM}$ $\mathrm{KPi}, \mathrm{pH} 7.8$, containing $5 \%$ glycerol was used. Concentrations of DTNB were $4 \int \mathrm{M}$ and $1.3 \mathrm{mM}$ DTNB, as indicated on results section. Both absorbance at $\sum_{412}$ and activities were measured at time intervals.

The amount of reacted sulfhydryl groups was calculated using $L_{412}=14150 \mathrm{M}^{-1} \mathrm{~cm}^{-1}$. Ellman (1959); Wright and Viola (1998) and Riddles et al. (1983). The activity was measured using the ferricenium assay.

\section{RESULT AND DISCUSSION}

Purification: IBD stability during purification was substantially increased by addition of $5 \mu \mathrm{M}$ DTT to all buffers used. This improves dramatically the yield of active IBD with ammonium sulfate cut-off and QSepharose chromatography steps. The reason for this was traced to an unexpectedly high reactivity of solvent accessible thiol groups of Cys residues. The purity of the isolated IBD was $>95 \%$.

Biochemical characterization: The subunit molecular weight obtained by MALDI-TOF spectroscopy is 42.689 $\mathrm{KDa} \pm 100 \mathrm{Da}$, which is comparable to calculated one (MW $=42.692 \mathrm{KDa}$ ) according to amino acid composition derived from the cDNA sequence. The oligomeric state was estimated by gel filtration and IBD found to be homotetramer with a $\mathrm{MW} \approx 170,000$ KDa (not shown). The extinction coefficient of the flavin absorption band in the visible spectrum (Fig. 1) was estimated to be $\approx 14.1 \mathrm{mM}^{-1} \mathrm{~cm}^{-1}$ using the SDS method (Whitby, 1953). This compares to $11.3 \mathrm{mM}^{-}$ ${ }^{1} \mathrm{~cm}^{-1}$ for free FAD (Lehman et al., 1990) and to values 12.3-14.8 for various ACADs; it is thus within the usual range for this family of flavoproteins.

The visible and ultraviolet absorbance spectrum of purified IBD is depicted in Fig. 1. Interestingly, pure IBD was obtained in a yellow form, although an associated green color can be noted during purification, which is gradually lost. This contrasts with some other ACADs that are obtained in "green forms" due to tightly bound CoA-persulfide (Engel and Massey, 1971). IBD spectrum is typical for other ACAD flavoproteins in having the two main bands centered on 450 and $370 \mathrm{~nm}$. Maxima absorbance ratio (268/444 $\mathrm{nm}$ ) for IBD found to be $\approx 5.4$ (Fig. 1), comparing to 5.7 for human MCAD (Kuechler et al., 1999).

Addition of the substrate isobutyryl-CoA under anaerobic conditions leads to formation of the reduced enzyme-2-methyl-acrylyl-CoA complex.

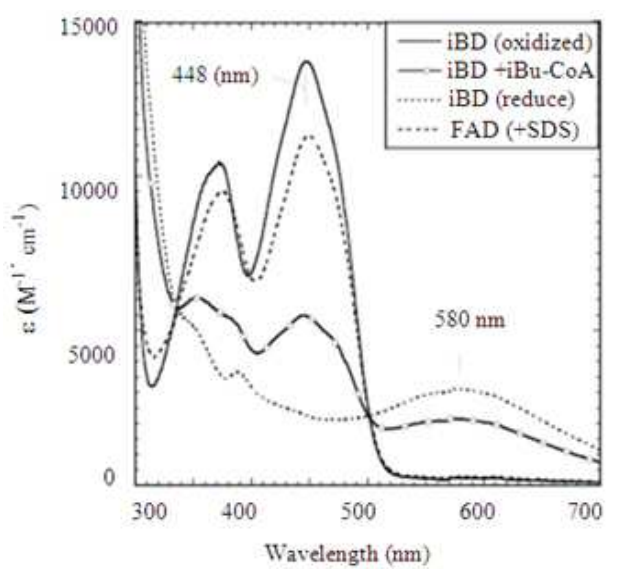

Fig. 1: (Ibrahim and Mohsen)

This is characterized by the decreasing of the absorption at $450 \mathrm{~nm}$ and formation of new maxima at $580-425 \mathrm{~nm}$ and a shoulder at $\approx 350 \mathrm{~nm}$. The latter two belong to the reduced flavin chromophore, while the long wavelength absorption is due to the chargetransfer interaction between the reduced flavin as donor and enoyl-CoA product as acceptor (Mueller, 1991). This band is characteristic for most ACADs, it varies depending on the ACAD and the bound product in its position and intensity (Finocchiaro et al., 1987) and can be used to identify the species (Fig. 1-3). IBD $\mathrm{pKa}$ value with isobutyryl-CoA was found to be 8.2 while the $\mathrm{pI}$ was estimated as $\approx 6.2$, value compares to 4.8 for MCAD (Coates and Tanaka, 1992).

Interaction with substrate analogs and inhibition: There are several substrate analogs that have gained prominence as inhibitors or inactivators for ACADs. Some of these were found to be useful for mechanistic studies (Schmidt et al., 1981). Methylenecyclopropylacetyl-CoA (cf. Structure in Fig. 4) which is naturally metabolite of hypoglycin and the causative agent of Jamaican vomiting sickness found to inactivate MCAD and SCAD via irreversible formation of covalent adducts (Wenz et al., 1981; Tanaka and Ikeda, 1990). None of these analogs listed in Fig. 4 found to inactivate IBD.

Reactivity of Cys thiol groups: Based on the observation that DTT substantially increases the stability of IBD, the role of 13 Cys residues in IBD tested using Ellman's reagent. Time dependence of the reaction of IBD with 4 folds and 100 folds molar excess of DTNB versus IBD activity is shown in Fig. 4. The data showed that, apparently, all 13 Cys residues present in IBD (extrapolated value for $\mathrm{T}_{\infty} \approx 12.7$ ) react at similar rates in monophasic processes. 
Am. J. Biochem. \& Biotech., 7 (2): 84-89, 2011

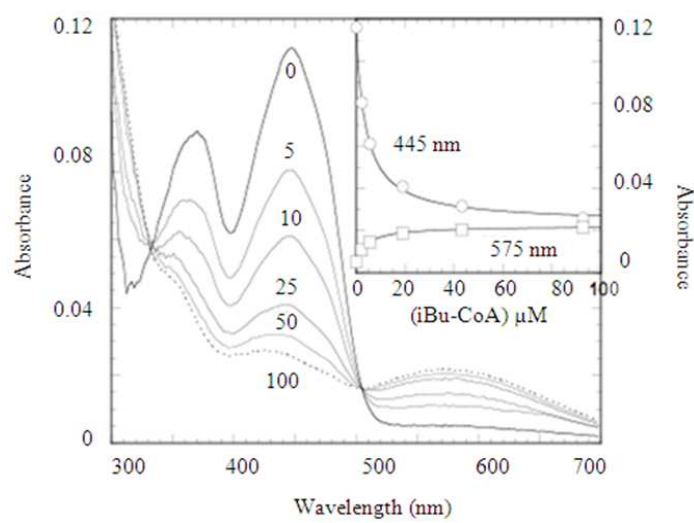

Fig. 2: (Ibrahim and Mohsen)

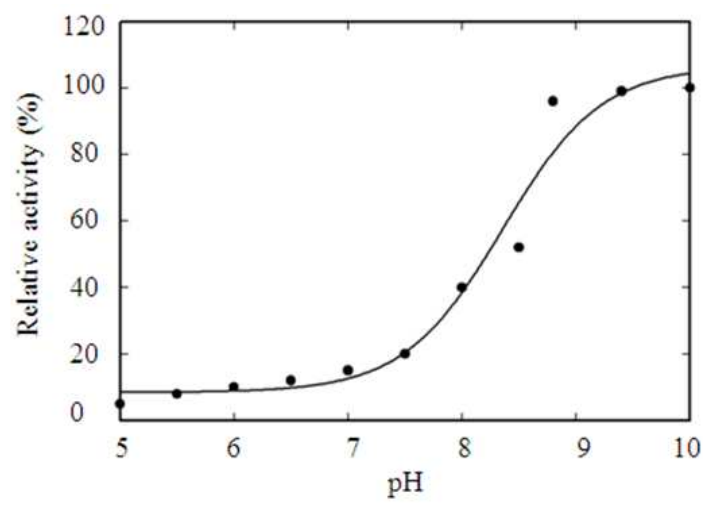

Fig. 3: (Ibrahim and Mohsen)

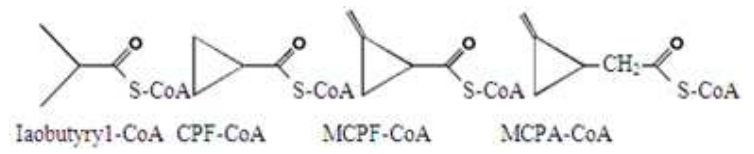

Fig. 4: (Ibrahim and Mohsen)

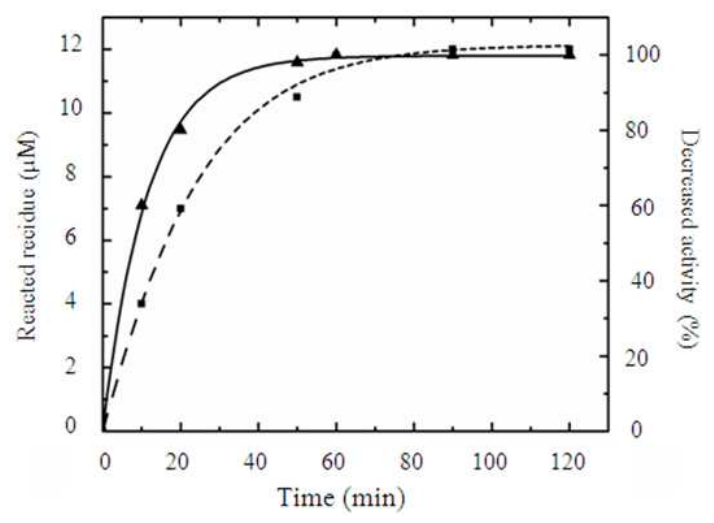

Fig. 5: (Ibrahim and Mohsen)
With 4 folds excess DTNB, 5 Cys residues reacted with DNTB decreasing the IBD activity by about $40 \%$ after the reaction with fourth residue (data not shown).

However, with 100 folds excess, almost all Cys residues reacted with DNTB and IBD activity decreased by $\approx 60 \%$ after the reaction with first four Cys residues and diminished completely diminished after reaction with 8-10 Cys residues (Fig. 5).

\section{CONCLUSION}

Upon the discovery of MCAD and SCAD by Boyer's group in the mid 60' the other members of the ACAD family were discovered in rather rapid succession (Boyer et al., 1964). However, only few years ago IBD was identified by Telford et al. (1999) and later, genetic defect in 2-years old child with reversible cardiomyopathy and deficiency in IBD activity in fibroblasts was identified (Sass et al., 2004).

IBD has relatively high $\mathrm{pI}$ (6.2) in comparison to values of other ACADs. However, it has $\mathrm{pKa} \approx 8.4$, similar to that of MCAD ( $\mathrm{pK} \approx 8.2)$ (Finocchiaro et al., 1987). Different types of ACADs inhibitors were examined for IBD inhibition. Acetyl-CoA and acetoacetyl-CoA are known to inhibit ACADs enzymes. With IBD, no inhibition found with either acetyl-CoA or acetoacetyl-CoA. Propionyl-CoA also known to inhibit both human and bovine SCAD (Coates and Tanaka, 1992; Ikeda ad Tanaka, 1983). On contrary, human IBD found to utilize propionyl-CoA as substrate. Interestingly, IBD not inhibited by cyclopropylformyl-CoA or its related compounds, that might be due to spatial configuration of these compounds to favorite substrate, isobutyryl-CoA.

IBD has the highest number of Cys residues, 13 residues, in all known ACADs, while there are 5 in SCAD, 7 in MCAD and 9 residues in IVD.

First trial of IBD purification without using DTT in purification buffers produced apoenzyme and IBD could not retained its activity upon FAD addition. It is well known that sulfhydril reagents inhibit ACADs enzymes (Coates and Tanaka, 1992; Ikeda andTanaka, 1983). We started to study the reasons of losing IBD activity and its FAD. We measured the reactivity and accessibility Cys residues by using $-\mathrm{SH}$ modification reagent DNTB. With 4 folds DTNB to IBD, the activity decreased by $40-50 \%$ by modification of 3-4 Cys residues.

The maximum number of Cys residues that can be modified at this DTNB conc. was about 5 residues. With higher DTNB conc., more than 100 folds, about $60-80 \%$ of the activity diminished upon modification of 4-7 Cys residues and completely abolished upon 
modification of the 10th residue (Fig. 5). These results suggest that Cys residue (s) are important for the proper structure, FAD and/or activity of human IBD in vivo. Our results consistent with previous reported results that showed presence of essential Cys residues located in the vicinity of the FAD- and substrate-binding sites within the active center of rat SCAD and MCAD enzymes (Okamura-Ikeda et al., 1985).

\section{ACKNOWLEDGEMENT}

Special thanks for Prof. Dr. Sandro ghisla, faculty of biology, konstanz university, germany, for his support and endless help for publishing this study.

\section{REFERENCES}

Battaile, K.P., T.V. Nguyen, J. Vockley and J.J.P. Kim, 2004. Structures of Isobutyryl-CoA dehydrogenase and enzyme-product complex: Comparison with isovaleryl- and short-chain acyl-coa dehydrogenases. J. Biol. Chem., 279: 1652616534. PMID: 14752098

Boyer, P.D., H. Lardy and K. Myrbäck 1964. The Enzymes: Prosthetic Groups and Cofactors, 2nd Edn., Academic Press, New York, pp: 497.

Coates, P.M. and K. Tanaka, 1992. New developments in fatty acid oxidation. Proceedings of the Second International Symposium on Clinical, Biochemical, and Molecular Aspects of Fatty Acid Oxidation, Held in Philadelphia, Pennsylvania, Wiley and Sons, New York, pp: 473-478. ISBN: 0471561444

Ellman, G.L., 1959. Tissue sulfhydryl groups. Arch. Biochem. Biophys., 82: 70-77. DOI: 10.1016/0003-9861(59)90090-6

Eminoglu, T.F., L. Tumer, I. Okur, F.S. Ezgu and G. Biberoglu et al., 2011. Very long-chain acyl CoA dehydrogenase deficiency which was accepted as infanticide. For. Sci. Int., 210: e1-e3. DOI: 10.1016/J.FORSCIINT.2011.04.003

Engel, P.C. and V. Massey, 1971. Green butyrylcoenzyme A dehydrogenase. An enzyme-acylcoenzyme A complex. Biochem. J., 125: 889-902. PMID: 5145911

Finocchiaro, G., M. Ito and K. Tanaka, 1987. Purification and properties of short chain acylCoA, medium chain acyl-CoA and isovaleryl-CoA dehydrogenases from human liver. J. Biol. Chem., 262: 7982-7989. PMID: 3597357

Ikeda, Y. and K. Tanaka, 1983. Purification and characterization of 2-methyl-branched chain acyl coenzyme A dehydrogenase, an enzyme involved in the isoleucine and valine metabolism, from rat liver mitochondria. J. Biol. Chem., 258: $9477-$ 9487. PMID: 6874697

Izai, K., Y. Uchida, T. Orii, S. Yamamoto and T. Hashimoto, 1992. Novel fatty acid beta-oxidation enzymes in rat liver mitochondria. I. Purification and properties of very-long-chain acyl-coenzyme A dehydrogenase. J. Biol. Chem., 267: 1027-1033. PMID: 1730632

Kuechler, B., A.G. Abdel-Ghany, P. Bross, A. Nandy and I. Rasched et al., 1999. Biochemical characterization of a variant human medium-chain acyl-CoA dehydrogenase with a disease-associated mutation localized in the active site. Biochem. J., 337: 225-230. PMID: 9882619

Lehman, T.C., D.E. Hale, A. Bhala and C. Thorpe, 1990. An acyl-coenzyme a dehydrogenase assay utilizing the ferricenium ion. Anal. Bioche., 186: 280-284. PMID: 2363500

Mann, M. and G. Talbo, 1996. Developments in matrixassisted laser desorption/ionization peptide mass spectrometry. Curr. Opin. Biotech., 7: 11-19. PMID: 8742374

Mayhew, S.G. and V. Massey, 1969. Purification and characterization of flavodoxin from peptostreptococcus elsdenii. J. Biol. Chem., 244: 794-802. PMID: 4976788

Mueller, F., 1991. Chemistry and Biochemistry of Flavoproteins. 1st Edn., CRC Press, Boca Raton, ISBN: 0849343941, pp: 520.

Oglesbee, D., M. He, N. Majumder, J. Vockley and A. Ahmad et al., 2007. Development of a newborn screening follow-up algorithm for the diagnosis of isobutyryl-CoA dehydrogenase deficiency. Genet. Med., 9: 108-116. PMID: 17304052

Okamura-Ikeda, K., Y. Ikeda and K. Tanaka, 1985. An essential cysteine residue located in the vicinity of the FAD-binding site in short-chain, mediumchain, and long-chain acyl-CoA dehydrogenases from rat liver mitochondria. J. Biol. Chem., 260: 1338-1345. PMID: 3968065

Riddles, P.W., R.L. Blakeley and B. Zerner, 1983. Reassessment of ellman's reagent. Meth. Enzymol., 91: 49-60. PMID: 6855597

Roe, C.R., S.D. Cederbaum, D.S. Roe, R. Mardach and A. Galindo et al., 1998. Isolated isobutyryl-CoA dehydrogenase deficiency: An unrecognized defect in human valine metabolism. Mol. Genet. Metab., 65: 264-271. PMID: 9889013

Sass, J.O., S. Sander and J. Zschocke, 2004. IsobutyrylCoA dehydrogenase deficiency: Isobutyrylglycinuria and ACAD8 gene mutations in two infants. J. Inherit. Metab. Dis., 27: 741-745. DOI: 10.1023/B:BOLI.0000045798.12425.1b 
Schmidt, J., J. Reinsch and J.T. McFarland, 1981. Mechanistic studies on fatty acyl-CoA dehydrogenase. J. Biol. Chem., 256: 11667-11670. PMID: 7298623

Tanaka, K. and Y. Ikeda, 1990. Hypoglycin and Jamaican vomiting sickness. Prog. Clin. Bio. Res., 321: 167-84. PMID: 2183231

Telford, E.R., L.M. Moynihan, A.F. Markham and N.J. Lench, 1999. Isolation and characterisation of a cDNA encoding the precursor for a novel member of the Acyl-CoA dehydrogenase gene family. Biochim. Bioph. Acta, 1446: 371-376. DOI: 10.1016/S0167-4781(99)00102-5

Wenz, A., C. Thorpe and S. Ghisla, 1981. Inactivation of general acyl-CoA dehydrogenase from pig kidney by a metabolite of hypoglycin A. J. Biol. Chem., 256: 9809-9812. PMID: 7275979
Whitby, L.G., 1953. A new method for preparing flavin-adenine dinucleotide. Bioche. J., 54: 437442. PMID: 13058921

Willard, J., C. Vicanek, K.P. Battaile, P.P. Van Veldhoven and A.H. Fauq et al., 1996. Cloning of a cDNA for short/branched chain acyl-coenzyme a dehydrogenase from rat and characterization of its tissue expression and substrate specificity. Arch. Biochem. Bioph., 331: 127-133. PMID: 8660691

Wright, S.K. and R.E. Viola, 1998. Evaluation of methods for the quantitation of cysteines in proteins. Anal. Bioch., 265: 8-14. PMID: 9866701

Ye, X., C. Ji, C. Zhou, L. Zeng and S. Gu et al., 2004. Cloning and characterization of a human cDNA ACAD10 mapped to chromosome 12q24.1. Mol. Biol. Rep., 3: 191-195. PMID: 15560374 